\title{
CONTENIDO INTERACTIVO CON H5P
}

\author{
Interactive content With H5p
}

\section{EPISTEMUS}

ISSN: 2007-8196 (electrónico)

ISSN: 2007-4530 (impresa)

Sergio Ramón Rossetti López ${ }^{1}$

Ma. Teresa García Ramirez ${ }^{2}$

Isaac Shamir Rojas Rodriguez ${ }^{3}$

Adelina Morita Alexander ${ }^{3}$

Alberto Olguín Moreno ${ }^{3}$

Recibido: 13 de marzo de 2019,

Aceptado: 10 de junio del 2019

Autor de Correspondencia:

Maestría. Sergio Ramón Rossetti López

Correo: sergio.rossetti@unison.mx

\section{Resumen}

En este artículo se presenta la herramienta digital H5P como alternativa para el diseño de materiales digitales interactivos de apoyo a la docencia. Se presentan dos alternativas para desarrollar Objetos Virtuales de Aprendizaje con H5P. Además, se muestran algunos otros beneficios que se obtienen al utilizar esta herramienta.

Palabras clave: Objetos de aprendizaje, sistema de gestión del aprendizaje, H5P.

\section{Abstract}

In this article the digital tool H5P is presented as an alternative for the design of interactive materials to support teaching. Two alternatives are presented to develop Virtual Learning Objects with H5P. In addition, some other benefits for this tool are shown.

Keywords: Learning object, learning managements system, H5P. 


\section{INTRODUCCIÒN}

En este trabajo se presenta una propuesta de herramienta innovadora para aquellos docentes que cuentan con conocimientos básicos en el manejo de software especializado y tengan interés en desarrollar sus propios recursos digitales de apoyo a la labor docente. H5P permite a cualquier usuario crear, editar y compartir sus propios Objetos Virtuales de Aprendizaje (OVA) y además, incorporarlos a sus cursos en los sistemas de gestión del aprendizaje. H5P es una plataforma open source que a través del navegador web, es posible crear este tipo de recursos digitales e interactivos a través de una interfaz muy sencilla y amigable. Además, H5P brinda una excelente alternativa para aquellos docentes que no cuenten con acceso a un sistema de gestión del aprendizaje y deseen incorporar a sus clases este tipo de recursos digitales.

\section{DESARROLLO}

La incorporación de las Tecnologías de la Información y de la Comunicación (TIC) en el ámbito de la educación han apoyado el proceso de enseñanza - aprendizaje, a la vez que han permitido trabajar con recursos digitales en Internet. Según los autores Baelo y Cantón [1] las TIC se han convertido definitivamente en un recurso que facilita el desarrollo de metodologías innovadoras en los procesos de enseñanza y aprendizaje.

Una forma de utilizar herramientas tecnológicas en el ámbito educativo a través de los llamados OVA, los cuales ayudan a promover el autoestudio, reforzar conocimientos vistos en clase y motivar el aprendizaje de los estudiantes [2].

Los OVA son recursos digitales que pueden ser utilizados como soporte para el aprendizaje y deben tener ciertas características como son: formato digital, propósito pedagógico, contenido interactivo y reusabilidad [7].

En la actualidad existen diversas herramientas digitales de apoyo a la enseñanza, que pueden ser muy complejas para su implementación, demasiado costosas y en ocasiones, incompatibles con los sistemas de gestión del aprendizaje.
En este sentido, $h 5 p$ es una herramienta innovadora para diseñar OVA, ya que permite crear materiales atractivos e interactivos a usuarios principiantes en el manejo de programación e informática. Además, H5P es una herramienta gratuita y de libre distribución con la cual un profesor de cualquier área del conocimiento pueda crear, compartir y reutilizar OVA.

Las siglas h5p son una abreviatura del término HTML5 y es una tecnología gratuita y abierta con licenciamiento de tipo Massachusetts Institute of Technology (MIT) de software libre. H5P permite diseñar más de 40 diferentes tipos de OVA y continuamente se adicionan nuevos tipos de contenidos para su utilización [x h5p]. Este tipo de materiales puede ser utilizado tanto para cursos online $o$ face2face.

Para conocer los diferentes tipos de contenidos interactivos, únicamente es necesario visitar el sitio web https://h5p.org, en el cual es posible visualizar ejemplos de OVA desarrollados con H5P y además, conocer sugerencias sobre cómo implementar determinado tipo de contenido en un curso.

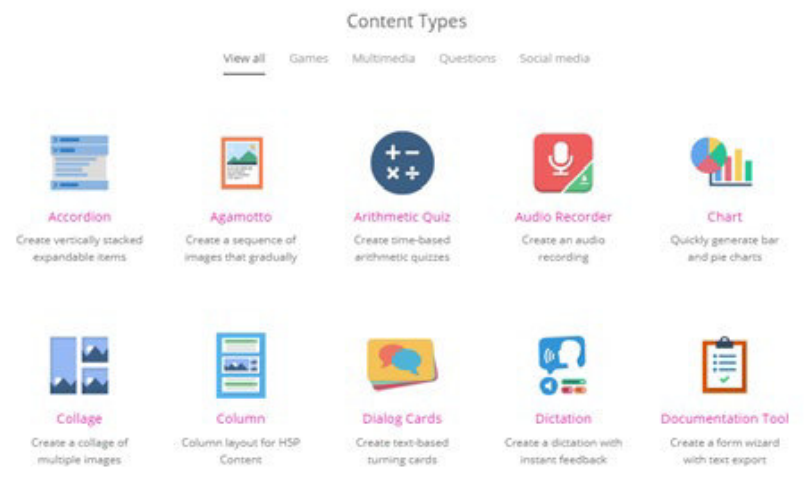

Figura 1. Contenido H5P.

\section{Implementación de H5P}

Existen dos alternativas disponibles para que los usuarios puedan utilizar el contenido interactivo de H5P y diseñar sus propios OVA. La primera alternativa es

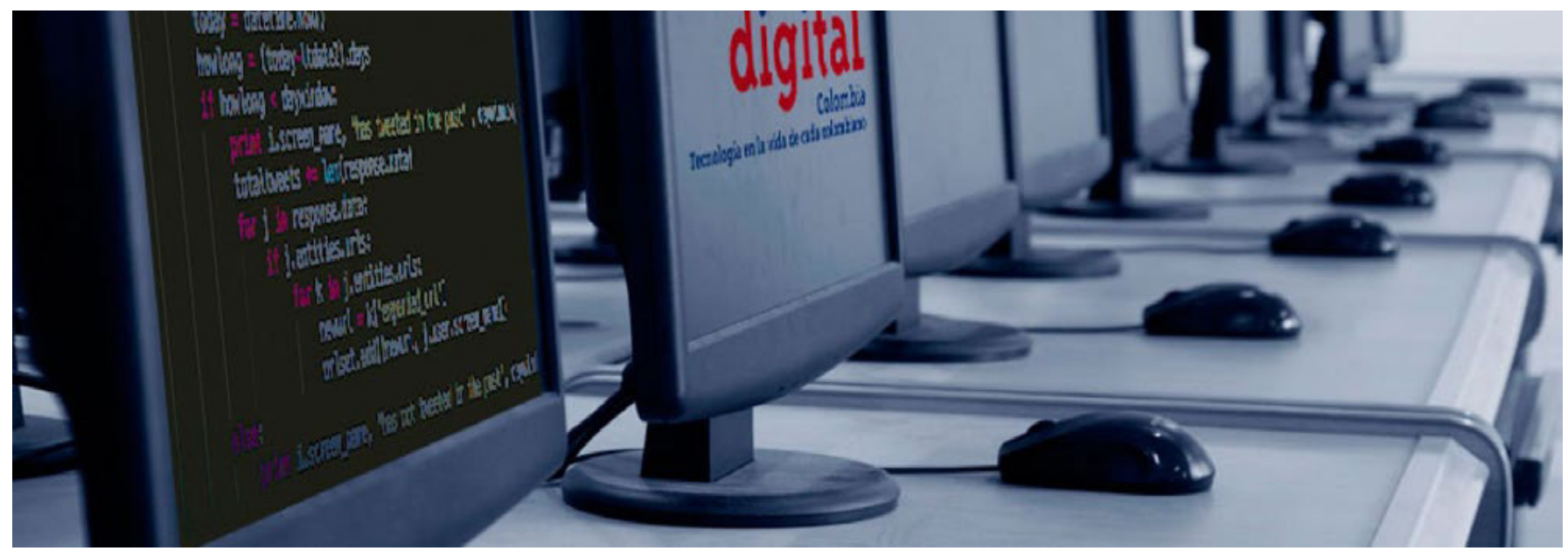




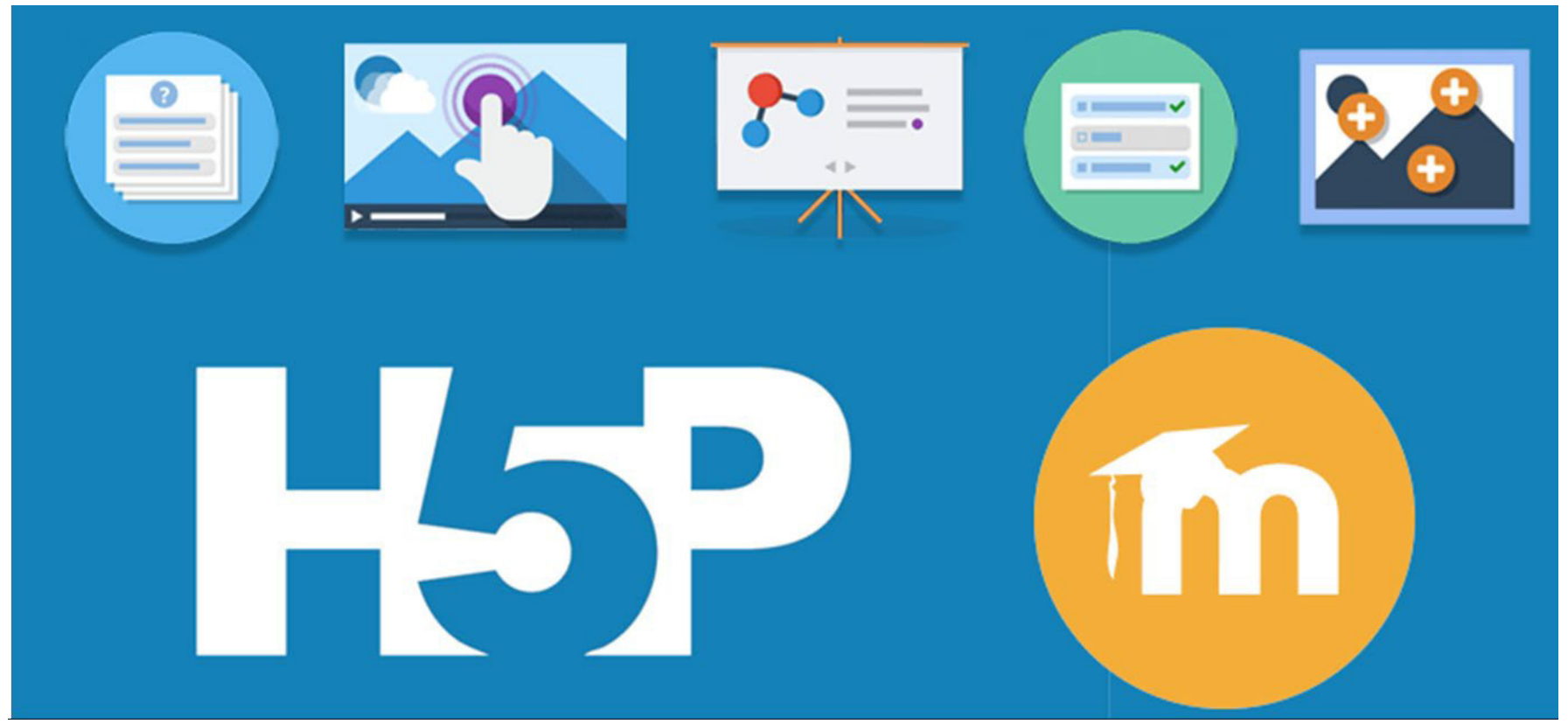

mediante el registro de usuario en el sitio web http://www. h5p.org. La segunda alternativa requiere de la autorización del administrador del sistema de gestión del aprendizaje donde se alojan los cursos del docente. En esta alternativa, es necesario realizar la instalación de un plugin para poder desarrollar OVA con H5P dentro del aula virtual del docente.

\section{Uso de H5P desde el sitio web}

Si no se cuenta a un sistema de gestión del aprendizaje, es posible iniciar con el desarrollo de material interactivo a través de la herramienta H5P Authoring tool. Para ello, es necesario crear una cuenta de usuario en el sitio web https://h5p.org/user/register.

Una vez registrado, podrá crear materiales interactivos a través de una herramienta guiada, intuitiva y fácil de utilizar. Algunos de los contenidos interactivos pueden ser

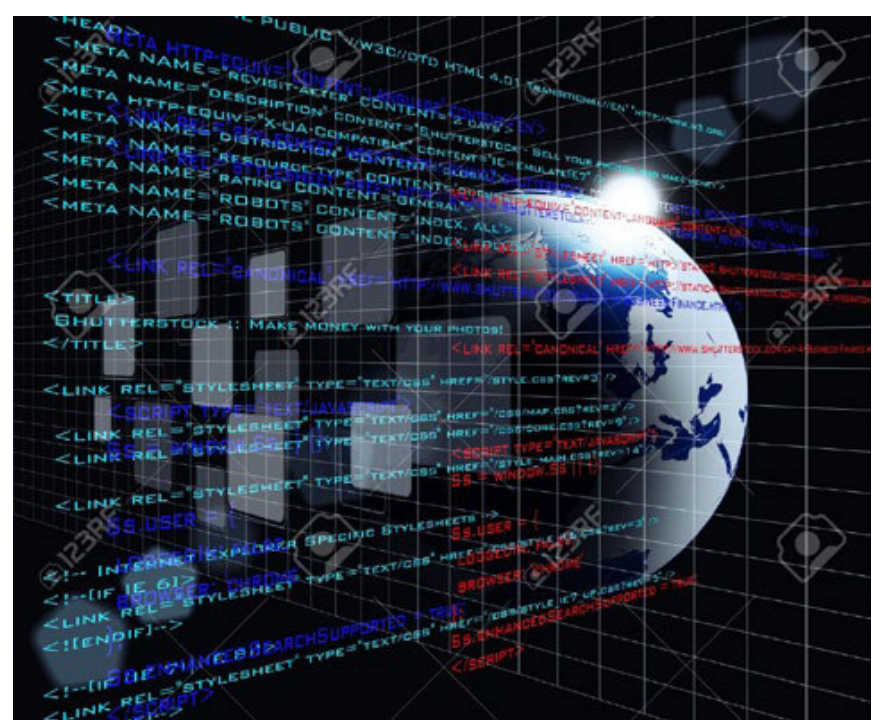

presentaciones online, videos interactivos, cuestionarios en línea, juegos, gráficos, entre otros [4].

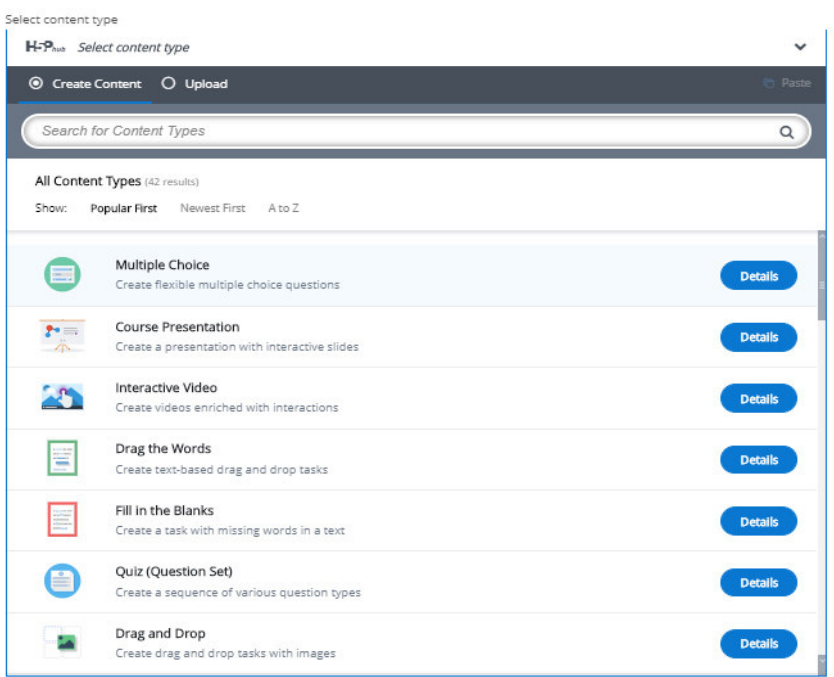

Figura 2. H5P Authoring tool.

Es importante mencionar que si se toma la decisión de utilizar el H5P Authoring tool, el OVA quedará publicado para toda la comunidad de usuarios H5P, a diferencia de utilizar el plugin para sistemas de gestión del aprendizaje.

Uso de H5P desde un sistema de gestión del aprendizaje

La segunda alternativa para la creación de contenido interactivo H5P es a través de un sistema de gestión del aprendizaje. Actualmente H5P cuenta con plugins integrar este tipo de contenidos a las plataformas Wordpress, Drupal y Moodle. Para poder realizar la integración de H5P con alguna de las estas plataformas, es necesario que el administrador del sistema realice la instalación del plugin de H5P en la plataforma. 
Una vez instalado el plugin, el docente podrá integrar contenido H5P en sus cursos, como si agregara cualquier recurso o actividad nativa de la plataforma [5]. En la siguiente imagen se puede observar un contenido H5P de tipo course presentation, integrado en un curso publicado en el sistema de gestión del aprendizaje Moodle.

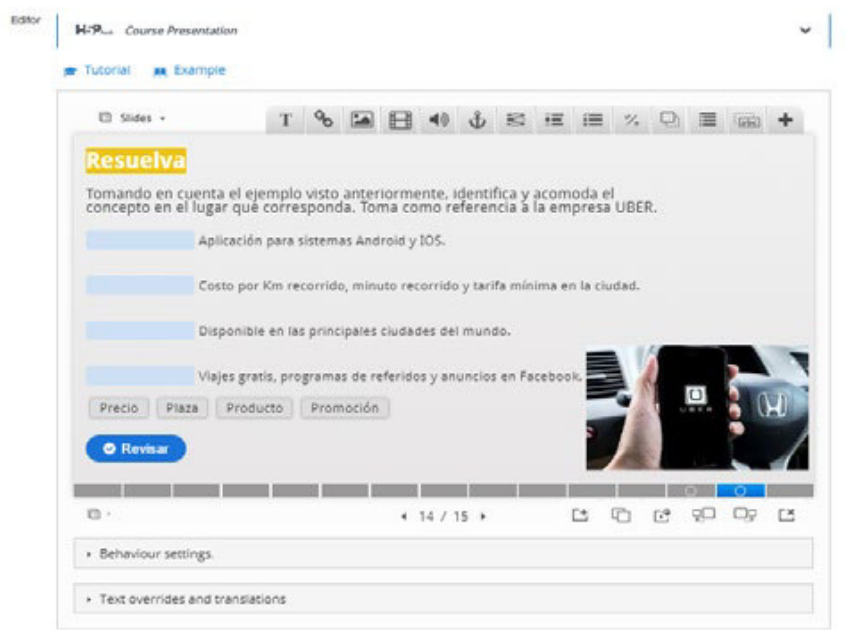

Figura 3. Contenido H5P en Moodle.

Cualquier usuario puede aprender a utilizar H5P en muy poco tiempo, debido a que es una herramienta que te lleva de la mano en cada paso. Además, H5P tiene la capacidad de generar contenido interactivo visualmente muy atractivo que supera a los recursos que tienen en general muchos sistemas de gestión del aprendizaje. Otra característica importante de H5P es la posibilidad de "reutilizar" los materiales, es decir, un docente puede compartir con colegas su materia y estos pueden adaptarlo, modificarlo y mejorarlo para utilizarlo en sus propios cursos.

\section{Finalmente, algunas de las razones más} importantes para utilizar H5P en sus cursos son:

1.H5P es una herramienta intuitiva. Hace algunos años se necesitaba de una persona con conocimientos de programación web para diseñar contenido interactivo. H5P permite a casi cualquier usuario diseñar contenido interactivo a través de un simple formulario.

2. H5P es compatible con múltiples plataformas. H5P puede ser utilizado en Moodle, Drupal y Wordpress y además, es compatible con cualquier dispositivo móvil.

3. La mayoría del contenido H5P puede ser utilizado por personas con alguna discapacidad, por ejemplo visual y auditiva. H5P cumple con los requerimientos WAI-ARIA (Accesible Rich Internet Aplications) para módulos dinámicos, navegación con teclado y subtítulos.

4. H5P es una tecnología open source. Cuando se utiliza H5P los usuarios pueden diseñar contenido interactivo [3], recibir asistencia de la comunidad en internet y colaborar con otros usuarios sin costo.

5. Para utilizar H5P no se requiere la instalación de algún complemento instalado en el navegador web del usuario o paquete de archivos [6]. A diferencia la herramienta SCORM, tradicionalmente utilizada para para la creación de OVA.

\section{CONCLUSIONES}

Con H5P es posible crear, editar y publicar OVA dinámicos, interactivos y visualmente muy atractivos, directamente en su navegador web preferido y desde cualquier dispositivo. Elegir el tipo de implementación para utilizar H5P dependerá si el docente tiene acceso a un sistema de gestión del aprendizaje con el plugin habilitado. De lo contrario, es posible utilizar la herramienta H5P Authoring tool con la cual, es posible crear contenido interactivo desde el sitio web de H5P para utilizarse como recurso en las clases. Además, es posible guardar los OVA en un archivo único con extensión .H5P para su edición o reutilización en otra plataforma. El contenido H5P cuenta con una gran cantidad de características personalizables, lo que lo hace una herramienta idónea para el desarrollo de OVA tanto para cursos online y bajo la modalidad tradicional face2face.

\section{BIBLIOGRAFÍA}

[1] R. Baelo y l. Cantón, "Use of Information and Communication Technologies in Castilla and León Universities". Comunicar, 18(35), 159-166.J. Clerk Maxwell, A Treatise on Electricity and Magnetism, 3rd ed., vol. 2. Oxford: Clarendon, 2010, pp.68-73.

[2] J. Barroso, J. Cabero, y A. Moreno. "La utilización de objetos de aprendizaje en Realidad Aumentada en la enseñanza de la medicina". Innoeduca. International Journal of Technology and Educational Innovation, 2016, vol. 2(2), pp.77.

[3] D. Gedera y A. Zalipour. "Use of interactive video for teaching and learning". In Australasian Society for Computers in Learning in Tertiary Education, ASCILITE, 2018, pp. 362-367.

[4] "H5P Documentation”, H5p.org, 2019. [Online]. Disponible en: https://h5p.org/.

[5] "Moodle plugins directory", Moodle.org, 2019. [Online]. Disponible en: https://moodle.org/plugins/.

[6] S. Rekhari y P. Sinnayah. "H5P and Innovation in Anatomy and Physiology Teaching". In Research and development in higher education:[re] valuing higher education: volume 41 : refereed papers from the 41st HERDSA Annual International Conference. 2-5 July 2018, Convention Centre, Adelaide, 2018, Vol. 41, pp. 191-205.

[7] D. Wiley. "The Learning Objects Literature". Handbook of Research on Educational Communications and Technology, 2007, vol.16, pp. 345-354.

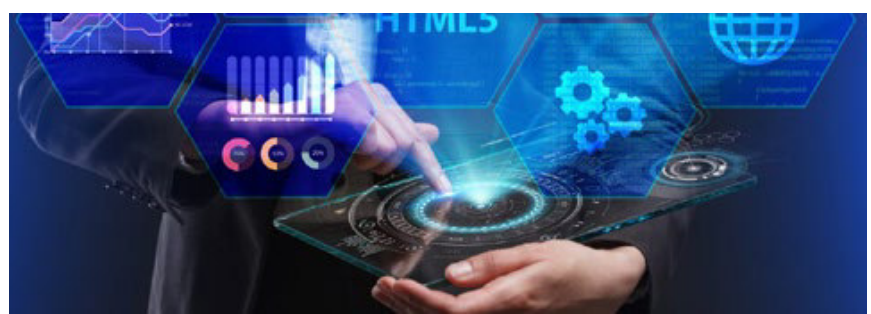

